DOI: 10.12957/demetra.2016.17431

\title{
Prevalência de anemia em gestantes acompanhadas nas unidades básicas de saúde do município de Caruaru-PE
}

\author{
Prevalence of anaemia in pregnant women attended in basic health units in Caruaru, \\ Pernambuco, Brazil
}

Weslla Karla Albuquerque Silva de Paula' Elaine Aparecida da Silva Gomes' Islane Carla da Silva'

${ }^{1}$ Faculdade ASCES. Associação Caruaruense de Ensino Superior e Técnico. Caruaru-PE, Brasil.

Correspondência / Correspondence Weslla Karla Albuquerque Silva de Paula wesllaalbuquerque@asces.edu.br

\section{Resumo}

Objetivo: Verificar a prevalência de anemia em gestantes acompanhadas nas unidades básicas de saúde do município de Caruaru, Pernambuco. Métodos: Estudo transversal, de natureza quantitativa, realizado a partir de dados secundários extraídos do banco de informações da pesquisa intitulada "Estado nutricional de gestantes: aspectos metodológicos epidemiológicos e implicações na assistência pré-natal". Realizou-se um recorte com as informações do referido município, sendo a amostra composta por 141 gestantes. A retirada de dados foi orientada por um instrumento contendo as variáveis de interesse do estudo, que envolviam aspectos sociodemográficos, antecedentes obstétricos e dados da gravidez atual. O desfecho "anemia" foi considerado como $\mathrm{Hb}<11 \mathrm{mg} / \mathrm{dl}$, segundo critérios adotados pela Organização Mundial da Saúde. Utilizou-se software Epi-info 7 para realizar as análises descritivas e bivariadas. Resultados: A prevalência de anemia encontrada foi de apenas $5 \%$. Maiores frequências de anemia foram observadas em gestantes adolescentes, com baixa escolaridade, pertencentes a menores classes econômicas, que realizaram apenas uma a duas consultas pré-natais, com início de acompanhamento no $2^{\circ}$ e $3^{\circ}$ trimestres e sem tratamento antianêmico na gestação atual. A análise bivariada não mostrou associação entre o desfecho "anemia" e as variáveis explanatórias avaliadas. Conclusão: Os achados desta pesquisa mostraram baixa prevalência de anemia pelos níveis de hemoglobina no município de Caruaru-PE, sugerindo a necessidade de novas investigações sobre os fatores envolvidos na determinação desta deficiência nutricional.

Palavras-chave: Anemia Ferropriva. Gravidez. Hemoglobinas. Deficiências Nutricionais. 


\section{Abstract}

Objective: To assess the prevalence of anemia in pregnant women attended in the basic health units in Caruaru, Pernambuco state, Brazil. Methods: Cross-sectional, quantitative study, conducted from secondary data drawn from the research information bank entitled "Nutritional status of pregnant women: methodological aspects and epidemiological implications for prenatal care." Information on that municipal district was extracted, and samples were composed fbyor 141 pregnant women. The data extracted was guided by an instrument containing the interest variables in the study, involvingd sociodemographic aspects, obstetric history and data from the current pregnancy. The outcome was considered as anemia $\mathrm{Hb}<11 \mathrm{mg} / \mathrm{dl}$, according to criteria adopted by the World Health Organization. We used Epi-info software 7 to make descriptive and bivariate analyses. Results: Prevalence of anemia was only $5 \%$. Higher frequencies of anemia were observed in pregnant adolescents, with low education, belonging to lower economic classes, who performed just 1-2 antenatal visits, starting this monitoring in the 2nd and 3rd quarters and without antianemic treatment during pregnancy. The bivariate analysis showed no association between the outcome anemia and evaluated explanatory variables. Conclusion: Research results showed low prevalence of anemia by hemoglobin levels in Caruaru, suggesting the need for further research on factors involving this nutritional deficiency.

Key words: Anemia. Pregnancy. Hemoglobins. Deficiency Diseases.

\section{Introdução}

A anemia constitui um importante problema de saúde pública com distribuição mundial, sendo mais prevalente em países menos desenvolvidos. Este agravo é resultado de ampla variedade de causas, mas a maior delas é a deficiência de ferro, o que contribui para que sejam frequentemente tratadas como sinônimos. ${ }^{1}$

Em 2008, a Organização Mundial da Saúde (OMS) publicou a estimativa global de anemia, que envolveu a compilação de dados de 93 países de 1993 a 2005, sendo demonstrado que 1,6 bilhão de pessoas (24,8\% da população mundial) são acometidas pela anemia, estimando-se uma prevalência dessa deficiência nutricional em mulheres grávidas na ordem de 41,8\%. Segundo a mesma publicação, a anemia em gestantes no Brasil apresentava-se como um problema de saúde pública de intensidade moderada, com frequência de $29,1 \%{ }^{2}$ 
No estado de Pernambuco, uma pesquisa que avaliou as condutas de serviços públicos de saúde em relação à anemia na gravidez verificou que das 440 gestantes que tinham registro da hemoglobina $(\mathrm{Hb})$ no cartão do pré-natal, $23 \%$ apresentavam valores $<11 \mathrm{~g} / \mathrm{dl} .{ }^{3} \mathrm{Na}$ capital do estado, pesquisa realizada no ambulatório de pré-natal do Instituto de Medicina Integral Prof. Fernando Figueira (IMIP) revelou frequência de anemia de 56,6\% nas gestantes acompanhadas naquele serviço. ${ }^{4}$

A anemia é uma das principais complicações da gravidez. ${ }^{5}$ Estima-se que, de cada dez gestantes que fazem o pré-natal, três são anêmicas. ${ }^{6}$ No ciclo gestatório, a anemia por carência de ferro pode estar associada ao aumento da mortalidade materna, fetal, perinatal, prematuridade, baixo-peso ao nascer e morbidade do infante. ${ }^{7}$

O referido agravo durante a gravidez destaca-se não só pela frequência com que se manifesta, mas também pelos efeitos nocivos à saúde da gestante e do concepto. ${ }^{8}$ Entre as principais causas da anemia em gestantes, destacam-se: baixo nível socioeconômico, maior número de partos, baixo nível educacional, reservas inadequadas de ferro, ausência de suplementação de ferro e dietas deficientes em ferro. ${ }^{9}$

A partir desta breve exposição, e tendo em vista que não há dados recentes publicados sobre a prevalência de anemia em gestantes no estado de Pernambuco e, por conseguinte, do município de Caruaru, tornou-se relevante o desenvolvimento desta pesquisa, considerando a magnitude da referida carência nutricional e seus efeitos deletérios à saúde da gestante e do concepto. Assim, a pesquisa objetivou verificar a prevalência de anemia em gestantes acompanhadas nas unidades básicas de saúde do município de Caruaru-PE.

\section{Métodos}

Estudo transversal, de natureza quantitativa, realizado a partir de dados secundários extraídos do banco de informações da pesquisa intitulada "Estado nutricional de gestantes: aspectos metodológicos epidemiológicos e implicações na assistência pré-natal”, estudo de prevalência, de base populacional, realizado no ano de 2012, através de iniciativa conjunta do Departamento de Nutrição da Universidade Federal de Pernambuco (UFPE) e do Instituto de Medicina Integral Prof. Fernando Figueira (IMIP), com apoio do Conselho Nacional de Pesquisa (CNPq), cujo objetivo foi descrever a situação nutricional de gestantes atendidas nos serviços de saúde do estado de Pernambuco. Participaram da pesquisa os municípios de Recife, Vitória de Santo Antão, Caruaru e Petrolina. A amostra geral foi constituída por 1.600 gestantes, sendo incluídas aquelas com idade entre 18 e 35 anos e idade gestacional entre oito e 32 semanas. 
Foram considerados critérios de exclusão: gestantes com dismorfias bem evidente (lordose e escoliose acentuadas), com história de aborto recente (menos de 08 semanas) e de sangramento em gestação que implicam tratamento hospitalar de pelo menos 24 horas, gestantes com cardiopatias descondensadas e com doenças renais (ureia sérica de $50 \mathrm{mg} / \mathrm{dl}$, creatinina acima de $1,6 \mathrm{mg} / \mathrm{dl}$ ). A amostragem foi não probabilística, por conveniência. Enquanto aguardavam a consulta pré-natal, as gestantes eram abordadas e convidadas a participar da pesquisa, sendo então assinado o Termo de Consentimento Livre e Esclarecido (TCLE). Os valores de hemoglobina foram retirados dos resultados de exames, obtidos por métodos automáticos de leitura da hemoglobina.

A pesquisa atual é um recorte dos dados referentes ao município de Caruaru-PE, no qual participaram 164 gestantes. Contudo, a atual amostra contou com 141 gestantes, uma vez que apenas estas possuíam resultado de hemograma. A retirada de informações no banco de dados foi realizada no mês de maio de 2014, mediante um instrumento contendo as variáveis de interesse. Essas envolviam aspectos sociodemográficos, antecedentes obstétricos e dados da gravidez atual. No estudo original, a variável "hemoglobina" foi verificada em sua forma contínua, contudo, nesta pesquisa foi transformada em uma variável dicotômica ( $\mathrm{Sim}-\mathrm{Hb}<11 \mathrm{mg} / \mathrm{dl}$; $\mathrm{Não}-\mathrm{Hb}>11 \mathrm{mg}$ / dl), segundo critérios adotados pela OMS. ${ }^{1}$

Para o presente estudo, a análise estatística foi realizada utilizando o software Epi-info 7, sendo desenvolvida em duas etapas: inicialmente foram descritas as características amostrais da população investigada. Para as variáveis contínuas, foram apresentadas as medidas de tendência central, e para as variáveis categóricas, suas frequências relativas e absolutas. Posteriormente, foi realizada análise bivariada com o objetivo de identificar as variáveis explanatórias que pudessem apresentar associação estatisticamente significante com a variável resposta $(p<0,05)$. A pesquisa foi aprovada pelo Comitê de Ética em Pesquisa da Faculdade ASCES (parecer no 659.161, em 23/05/14).

\section{Resultados}

Neste estudo, foram analisadas as informações das 141 gestantes com registro do valor da hemoglobina, as quais compuseram o banco de dados da pesquisa original. A prevalência de anemia encontrada foi de apenas $5 \%$ (gráfico 1). 


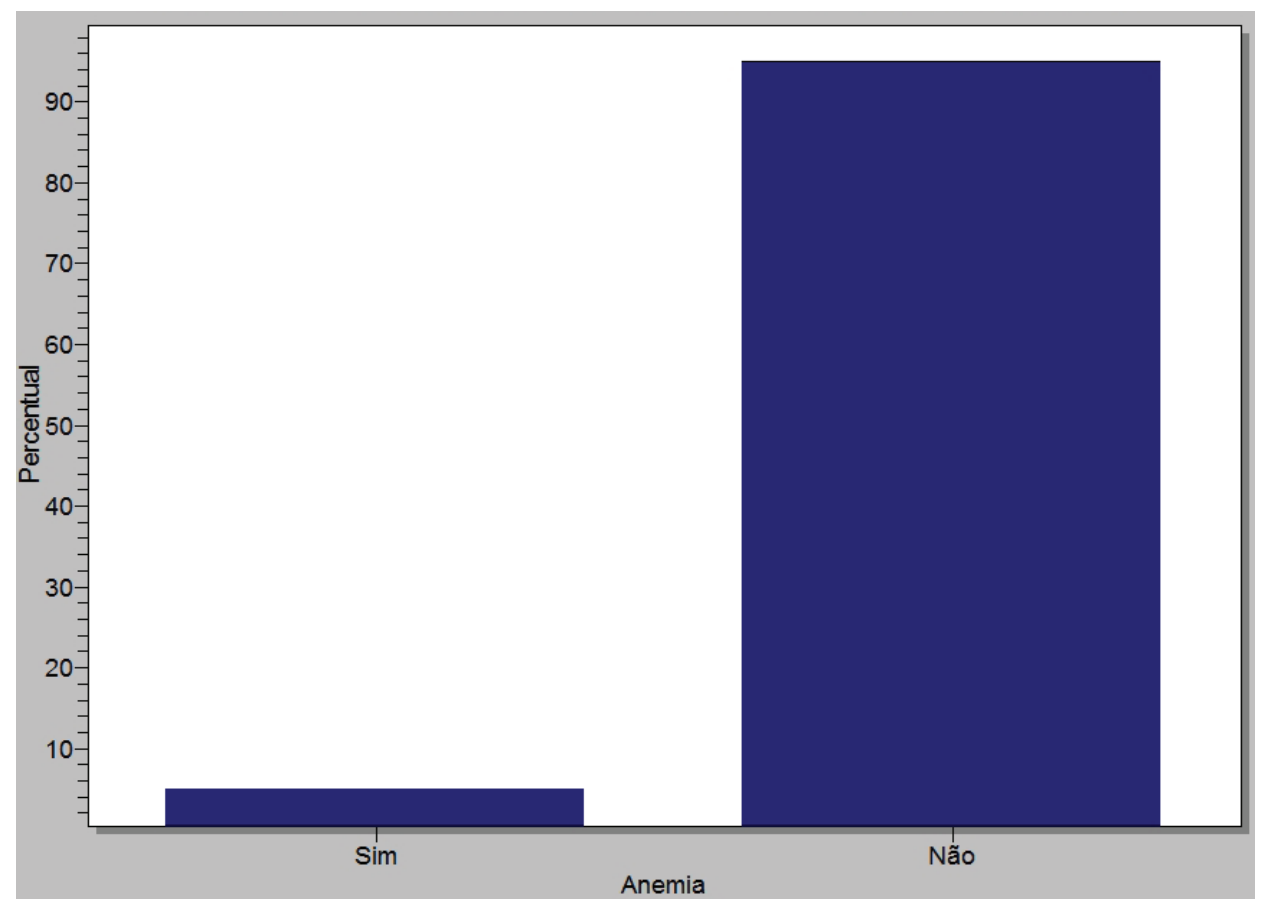

Gráfico 1. Prevalência de anemia em gestantes assistidas em Unidades Básicas de Saúde. Caruaru-PE, 2012.

As informações quanto às características demográficas, socioeconômicas e relativas à saúde das gestantes encontram-se sumarizadas na tabela 1 .

Tabela 1. Prevalência de anemia em gestantes assistidas pelas UBS, segundo características demográficas, socioeconômicas e relativas à saúde. Caruaru-PE, 2012.

\begin{tabular}{|c|c|c|c|c|c|c|}
\hline \multirow{3}{*}{ Variáveis } & \multirow{3}{*}{$\mathbf{N}$} & \multicolumn{4}{|c|}{ Anemia } & \multirow{3}{*}{$p$} \\
\hline & & \multicolumn{2}{|c|}{ Sim } & \multicolumn{2}{|c|}{ Não } & \\
\hline & & $\mathbf{N}$ & $\%$ & $\mathbf{n}$ & $\%$ & \\
\hline Idade & & & & & & 0,3156 \\
\hline$<20$ anos & 16 & 2 & 12,5 & 14 & 87,5 & \\
\hline 20 a 29 anos & 90 & 4 & 4,4 & 86 & 95,6 & \\
\hline$\geq 30$ anos & 35 & 1 & 2,9 & 34 & 97,1 & \\
\hline
\end{tabular}




\begin{tabular}{|c|c|c|c|c|c|c|}
\hline \multirow{3}{*}{ Variáveis } & \multirow{3}{*}{$\mathbf{N}$} & \multicolumn{4}{|c|}{ Anemia } & \multirow{3}{*}{$p$} \\
\hline & & \multicolumn{2}{|c|}{ Sim } & \multicolumn{2}{|c|}{ Não } & \\
\hline & & $\mathbf{N}$ & $\%$ & $\mathbf{n}$ & $\%$ & \\
\hline Cor & & & & & & 0,5154 \\
\hline Branca & 63 & 2 & 3,2 & 61 & 96,8 & \\
\hline Parda & 47 & 2 & 4,3 & 45 & 95,7 & \\
\hline Negra & 14 & 1 & 7,1 & 13 & 92,9 & \\
\hline Outro & 17 & 2 & 11,8 & 15 & 88,2 & \\
\hline Renda $\dagger$ & & & & & & 0,5843 \\
\hline$<0,5 \mathrm{SM}$ & 13 & 1 & 7,7 & 12 & 92,3 & \\
\hline $0,5-<1 \mathrm{SM}$ & 47 & 2 & 4,3 & 45 & 95,7 & \\
\hline $1-<2 \mathrm{SM}$ & 38 & 3 & 7,9 & 35 & 92,1 & \\
\hline$\geq 2 \mathrm{SM}$ & 20 & - & - & 20 & 100 & \\
\hline Classe econômica & & & & & & 0,0657 \\
\hline B & 21 & - & - & 21 & 100 & \\
\hline $\mathrm{C}$ & 72 & 2 & 2,8 & 70 & 97,2 & \\
\hline $\mathrm{D}$ & 28 & 4 & 14,3 & 24 & 85,7 & \\
\hline $\mathrm{E}$ & 2 & - & - & 2 & 100 & \\
\hline Escolaridade (anos de estudo) & & & & & & 0,1808 \\
\hline Nenhum & 1 & - & - & 1 & 100 & \\
\hline $1-9$ & 52 & 5 & 9,6 & 47 & 90,4 & \\
\hline $10-12$ & 68 & 1 & 1,5 & 67 & 98,5 & \\
\hline$\geq 13$ & 8 & - & - & 8 & 100 & \\
\hline № de pessoas no domicílio & & & & & & $0,6558^{\#}$ \\
\hline $1-3$ & 81 & 4 & 4,9 & 77 & 95,1 & \\
\hline $4-8$ & 43 & 2 & 4,7 & 41 & 95,3 & \\
\hline № de crianças $<5$ anos & & & & & & $0,5706^{\#}$ \\
\hline Nenhuma & 94 & 5 & 5,3 & 89 & 94,7 & \\
\hline$\geq 1$ & 47 & 2 & 4,3 & 45 & 95,7 & \\
\hline
\end{tabular}




\begin{tabular}{|c|c|c|c|c|c|c|}
\hline \multirow{3}{*}{ Variáveis } & \multirow{3}{*}{$\mathbf{N}$} & \multicolumn{4}{|c|}{ Anemia } & \multirow{3}{*}{$p$} \\
\hline & & \multicolumn{2}{|c|}{ Sim } & \multicolumn{2}{|c|}{ Não } & \\
\hline & & $\mathbf{N}$ & $\%$ & n & $\%$ & \\
\hline № de gravidez & & & & & & $0,5904^{\#}$ \\
\hline $1-2$ & 104 & 5 & 4,8 & 99 & 95,2 & \\
\hline$\geq 3$ & 37 & 2 & 5,4 & 35 & 94,6 & \\
\hline Última gestação ( em anos) & & & & & & $0,6244^{\#}$ \\
\hline Até 2 anos & 22 & 1 & 4,5 & 21 & 95,5 & \\
\hline$>2$ anos & 58 & 2 & 3,4 & 56 & 96,6 & \\
\hline $\begin{array}{l}\text { N de consultas pré-natal } \\
\text { (gravidez atual) }\end{array}$ & & & & & & $0,2767^{\#}$ \\
\hline $1-2$ & 75 & 5 & 6,7 & 70 & 93,3 & \\
\hline$\geq 3$ & 66 & 2 & 3,0 & 64 & 97,0 & \\
\hline $\begin{array}{l}\text { Inicio do Pré-natal ( por } \\
\text { trimestre) }\end{array}$ & & & & & & $0,2345^{\#}$ \\
\hline $1^{\mathrm{o}}$ trimestre & 114 & 4 & 3,5 & 110 & 96,5 & \\
\hline $2^{\circ}-3^{\circ}$ Trimestre & 21 & 2 & 9,5 & 19 & 90,5 & \\
\hline $\begin{array}{l}\text { Tratamento antianêmico } \\
\text { (gestação atual) }\end{array}$ & & & & & & 0,0822 \\
\hline Sim & 108 & 3 & 2,8 & 105 & 97,2 & \\
\hline Não & 32 & 4 & 12,5 & 28 & 87,5 & \\
\hline Não sabe & 1 & - & - & 1 & 100 & \\
\hline $\begin{array}{l}\text { Suplemento vitamínico } \\
\text { (gestação atual) }\end{array}$ & & & & & & 0,7845 \\
\hline Sim & 27 & 2 & 7,4 & 25 & 92,6 & \\
\hline Não & 106 & 5 & 4,7 & 101 & 95,3 & \\
\hline Não sabe & 3 & - & - & 3 & 100 & \\
\hline
\end{tabular}

$\uparrow$ A variável renda familiar foi tomada em sua forma contínua, sendo posteriormente categorizada considerando-se o valor do salário mínimo de $\mathrm{R} \$ 724,00$. \# Considerou-se o valor de p do teste exato de Fisher. 
A média de idade das gestantes estudadas foi de 25,6 anos, sendo a faixa etária de menores de 20 anos aquela com maior frequência de gestantes anêmicas (12,5\%). Os resultados mostraram maior prevalência de anemia em mulheres que referiram possuir cor negra (7,1\%). As mulheres pertencentes à classe econômica D apresentaram maiores prevalências de anemia (14,3\%). Aproximadamente $8 \%(\mathrm{n}=3)$ da amostra possuíam renda familiar entre um e dois salários mínimos.

Em relação à escolaridade, observaram-se maiores prevalências de anemia entre as gestantes que possuíam escolaridade inferior a nove anos de estudo constituindo 9,6\% ( $n=5)$ da amostra.

No que diz respeito ao número de pessoas por domicílio, não houve diferença significativa $(p>$ $0,05)$ na prevalência de anemia em gestantes (1-3 pessoas: 4,9\%; $\geq 4$ pessoas: $4,7 \%$ ). Foi observada maior deficiência de ferro entre as gestantes que não tinham nenhuma criança menor de cinco anos (5,3\%), bem como nas gestantes que referiram três ou mais gestações $(5,4 \%)$.

Verificou-se que a prevalência de anemia diminuiu conforme o intervalo interparto. Entre as gestantes que tinham intervalo até dois anos, 4,5\% ( $\mathrm{n}=1)$ apresentavam-se anêmicas, número diminuiu quando o intervalo interpartal foi maior que dois anos $(3,4 \%)$. No que se refere ao número de gravidez, $5,4 \%$ das anêmicas eram multíparas com três ou mais gestações. Em relação ao número de consultas, destaca-se que das gestantes que realizaram de uma a duas consultas, $6,7 \%(n=5)$ expressaram anemia.

Em média, as 141 gestantes amostradas estavam com pouco mais de 19 semanas (19,4 semanas), sendo que aproximadamente $31 \%$ dessas mulheres apresentavam até 12 semanas quando do momento da abordagem pelo entrevistador. Das gestantes que iniciaram o pré-natal no segundo e terceiro trimestre, $9,5 \%(\mathrm{n}=2)$ possuíam valor de hemoglobina menor que $11 \mathrm{mg} / \mathrm{dl}$.

Quanto ao tratamento antianêmico, a pesquisa identificou que das 108 que utilizavam suplementação de ferro apenas 2,8\% (n=3) eram consideradas anêmicas, número que atinge proporções mais elevadas quando consideradas as mulheres que não faziam tratamento $12,5 \%$ ( $\mathrm{n}=4$ ).

Ao se realizar a análise bivariada, verificou-se que não houve associação estatisticamente significante entre as variáveis explanatórias avaliadas e o desfecho "anemia”, uma vez que nenhuma variável apresentou valor de $\mathrm{p}<0,05$.

\section{Discussão}

A prevalência encontrada neste estudo foi inferior ao índice considerado de importância alarmante pela OMS. Pode-se classificar a significância populacional da prevalência de anemia como normal ou aceitável (abaixo de 5\%), leve (de 5 a 19,9\%), moderada (de 20 a 39,9\%) e grave (maior ou igual a 40\%). ${ }^{2}$ Dessa forma, a frequência observada desse agravo (5\%) a qualifica como leve problema de saúde pública na população estudada, conforme demostrado no gráfico 1. Este 
valor é semelhante ao encontrado em estudo realizado por Camargo et al., ${ }^{10}$ onde $4,8 \%$ das gestantes encontravam-se anêmicas, considerando o ponto de corte $\mathrm{Hb}<1 \mathrm{lg} / \mathrm{dl}$.

A hemoglobina é o único parâmetro rotineiramente utilizado na rede pública de assistência pré-natal, sugerindo que a maioria das gestantes com deficiência de ferro não é identificada. ${ }^{10} \mathrm{~A}$ concentração de hemoglobina reflete uma situação de deficiência nutricional já estabelecida. ${ }^{5}$ Nas gestantes, o nível de hemoglobina deve ser analisado com cuidado, pois as modificações fisiológicas na composição sanguínea durante a gravidez, como a hemodiluição, dificultam o reconhecimento da condição patológica. ${ }^{5}$

Quando se analisaram os casos de anemia por faixa etária, observou-se maior ocorrência em gestantes com menos de 20 anos. Este resultado foi similar ao encontrado por Tapia et al..$^{11} \mathrm{em}$ estudo realizado no Município de Belo Horizonte-MG, no qual maiores índices de anemia foram encontrados em adolescentes grávidas menores de 18 anos (14,02\%), o que pode ser explicado pela maior necessidade de ferro para atender às demandas do crescimento próprias da idade.

Os resultados mostraram que a anemia foi mais prevalente em mulheres negras. Esse resultado converge com aquele observado por Fabian et al., ${ }^{12} \mathrm{em}$ que o risco de anemia nas mulheres negras manteve-se três vezes maior do que nas brancas. A diferença étnica na prevalência de anemia também foi evidenciada na Pesquisa Nacional de Demografia e Saúde (PNDS). ${ }^{13}$

No presente estudo, observou-se que a maioria das gestantes anêmicas pertencia à classe econômica D e possuía renda menor que dois salários mínimos. Alguns autores enfatizam que os riscos para instalação de deficiências nutricionais ocorrem principalmente nas classes sociais menos favorecidas, cujo consumo de alimentos, na maioria das vezes, é inadequado. ${ }^{11,14}$

Investigação realizada por Ferreira et al. ${ }^{15}$ com gestantes da região semiárida de Alagoas constatou que o baixo nível socioeconômico estava relacionado a uma maior frequência de anemia. A baixa renda acarreta menor poder de aquisição de alimentos considerados fonte de ferro de alta biodisponibilidade (carnes, aves, peixes e miúdos) e tal fato contribuiria para o aumento da anemia.

Quanto à escolaridade, a maioria das gestantes anêmicas possuía escolaridade inferior a nove anos de estudo. Segundo Santos e Cerqueira, ${ }^{8}$ a maior ocorrência de anemia e de outras deficiências nutricionais decorre do nível educacional e cultural dos indivíduos. Há evidências de que gestantes com menor grau de escolaridade apresentam prevalências mais elevadas de anemia..$^{9,16,17}$

O fato de não terem sido observadas diferenças significativas na prevalência de deficiência de ferro entre as gestantes, segundo número de pessoas residentes no domicílio, assemelha-se aos achados de outros autores. ${ }^{8,17}$ Por sua vez, a prevalência de anemia foi maior entre as gestantes que não tinham nenhuma criança com idade menor de cinco anos, discordando de outros estudos realizados em cidades nordestinas, que demostraram maior prevalência de anemia em gestantes com até dois filhos. ${ }^{14,17}$ Hipoteticamente, as gestantes participantes da presente pesquisa podem ter sido 
expostas a outros fatores que determinam anemia, a exemplo de abortamento e ingesta insuficiente de alimentos ricos em ferro, mas por essas variáveis não terem sido aferidas, não se pode afirmar.

A frequência de anemia aumentou conforme o número maior de gestação, mas não houve associação estatisticamente significante entre essas variáveis, corroborando os resultados observados em gestantes do semiárido alagoano ${ }^{15}$ e em grávidas de um município do interior do Rio Grande do Sul. ${ }^{9}$ Sabe-se que a gestação e as perdas sanguíneas decorrentes do parto imprimem à mulher maior necessidade de ferro para compensar os menores níveis de hemoglobina impostos por esses eventos.

A associação entre o menor intervalo interpartal e a presença de baixos níveis de hemoglobina nas gestantes foi demonstrada nesta pesquisa. Este resultado se contrapõe aos achados de Santos et al., ${ }^{14}$ os quais relatam frequência similar de anemia, tanto para as gestantes cujo intervalo de parto foi menor que 24 meses, quanto para aquelas cujo intervalo interpartal foi maior. É aconselhável que o intervalo entre as gestações seja de, no mínimo, dois anos. ${ }^{18}$

Estudo realizado por Miglioli et al. ${ }^{17}$ no estado de Pernambuco, o qual analisou a ocorrência de anemia em crianças menores de cinco anos e mulheres no período reprodutivo, constatou que esta deficiência nutricional foi mais prevalente no grupo de gestantes com três ou menos consultas durante a gravidez, corroborando os achados do presente estudo. O Ministério da Saúde preconiza que o total de consultas pré-natais seja de, no mínimo, seis..$^{18}$

Esta pesquisa apontou aumento na prevalência de anemia de acordo com a evolução da gestação, informações convergentes com as encontradas em estudo realizado por Totti et al., ${ }^{19}$ no qual se observou anemia no primeiro trimestre em 2,8\% das gestantes, sendo menos frequente que no segundo $(7,14 \%)$ e terceiro trimestres de gestação $(9,6 \%)$. Fato semelhante foi visto em pesquisa realizada por Rocha et al. ${ }^{16}$ no município de Viçosa-MG, onde a prevalência dessa deficiência nutricional evoluiu conforme o avanço da idade gestacional. As necessidades de ferro variam, acentuadamente, a cada trimestre gestacional. No primeiro trimestre, os requerimentos não se alteram..$^{20}$ Entretanto, a partir do segundo, esses requerimentos começam a se elevar, em decorrência do aumento das necessidades de oxigênio para mãe e o feto, perdurando até o final da gestação. ${ }^{21}$

A maioria das gestantes avaliadas nesta pesquisa fazia uso de medicamento antianêmico. Este resultado é semelhante ao realizado no município de Feira de Santana-BA, por Santos e Cerqueira, ${ }^{8}$ segundo o qual 78,5\% das gestantes faziam uso de medicamento antianêmico. No presente estudo, verificou-se que 12,5\% das gestantes acometidas por essa deficiência nutricional referiram não utilizar o suplemento. A suplementação de ferro é universalmente recomendada durante a gestação, ${ }^{9}$ mesmo na ausência de anemia, objetivando satisfazer o aumento dos requerimentos desse mineral durante os dois últimos trimestres gestacionais. ${ }^{20,21}$ 
A despeito das limitações metodológicas do presente estudo, o pequeno tamanho da amostra e a baixa prevalência de anemia percebida podem ter contribuído para não terem sido observadas associações estatisticamente significantes entre anemia e variáveis sabidamente conhecidas como concorrentes para a ocorrência deste desfecho. Ademais, por não ter sido realizada a análise multivariada, estes achados podem ter sofrido influência de algum fator de confusão não identificado na análise bivariada. Sabe-se também que trabalhar com banco de dados restringe a investigação às variáveis disponíveis, impossibilitando a verificação de outros fatores contribuintes para o evento.

\section{Conclusão}

Os achados desta pesquisa mostraram baixa prevalência de anemia pelos níveis de hemoglobina no município de Caruaru-PE, não sendo encontrados fatores estatisticamente associados ao referido desfecho.

Na pesquisa, não se chega a todas as respostas que seriam desejáveis, mas foi possível realizar um dimensionamento inicial da amplitude da anemia em um estrato geográfico caracterizado por contrastes ambientais e sociais. Desse modo, sugere-se a necessidade de novas investigações sobre os fatores envolvidos na determinação desta deficiência nutricional.

\section{Referências}

1. World Health Organization. Iron deficiency anaemia: assessment, prevention and control: a guide for programme managers. Geneva: WHO; 2001. 114 p.

2. World Health Organization. Worldwide prevalence of anaemia 1993 2005: WHO global database on anaemia. Geneva: WHO; 2008. 40 p.

3. Santos ICRV, Batista Filho M. Anemia no atendimento pré-natal em Pernambuco: avaliação de condutas preventivas e curativas em serviços públicos de saúde. Recife: IMIP: 2001. (Publicações Científicas do Instituto Materno Infantil de Pernambuco, IMIP, n. 5).

4. Bresani CC, Souza BAI, Batista Filho M, Figueiroa JN. Anemia e ferropenia em gestantes: dissensos de resultados de um estudo transversal. Bras. Saúde Mater. 2007; (Suppl. 1): s15-s21.

5. Costa CM, Brum IR, Lima ES. Anemia e marcadores séricos da deficiência de ferro em grávidas atendidas na rede pública municipal de Manaus, Amazonas, Brasil. Acta Amaz. 2009; 39(4):901-905.

6. Brasil. Ministério da Saúde. Secretaria de Atenção à Saúde. Política nacional de alimentação e nutrição. 2. ed. Brasília: Ministério da Saúde; 2003.

7. Batista Filho M, Souza AI, Bresani CC. Anemia como problema de saúde pública: uma realidade atual. Ciênc. Saúde Coletiva 2008; 13(6):1917-1922. 
8. Santos PNP, Cerqueira EMM. Prevalência de anemia nas gestantes atendidas em unidades de Saúde em Feira de Santana, Bahia, entre out. 2005 e mar. 2006. Rev. Brasileira de Análises Clínicas 2008; 40(3):219-223.

9. Vitolo MR, Boscaini C, Bortolini GA. Baixa escolaridade como fator limitante para o combate à anemia entre gestantes. Rev. Bras. Ginecol. Obstet. 2006; 28(6):331-339.

10. Camargo RMS, Espinosa MM, Pereira SF, Schirmer. Prevalência de anemia e deficiência de ferro: relação com índice de massa corporal em gestantes do Centro - Oeste do Brasil. Medicina (Ribeirão Preto) 2013; 46(2):118-127.

11. Tapia MEL, Ro MLSF, Gontijo MLBZ, Martins CS, Viana KA, Dusse LMS, et al. Prevalência de anemia nas gestantes atendidas no Sistema Único de Saúde - Secretaria Municipal de Saúde - Prefeitura de Belo Horizonte. Rev. Bras. Anal. Clin. 2010; 42(4):277-281.

12. Fabian C, Olinto MTA, Dias-da-Costa JS, Bairros F, Nácul LC. Prevalência de anemia e fatores associados em mulheres adultas residentes em São Leopoldo, Rio Grande do Sul, Brasil. Cad. Saúde Pública 2007; 23(5):1199-1205.

13. Centro Brasileiro de Análise e Planejamento. Pesquisa Nacional de Demografia e Saúde da Criança e da Mulher - PNDS 2006: dimensões do processo reprodutivo e da saúde da criança. Brasília: Cebrap; 2009. Série G. Estatística e Informação em Saúde.

14. Santos FF, Conceição SIO, Monteiro SG. Anemia em gestantes atendidas em maternidades, em São Luís (MA). Cad. Pesq. 2012; 19(2):54-61.

15. Ferreira HDS, Moura FA, Cabral Junior CR. Prevalência e fatores associados à anemia em gestantes da região semi-árida do Estado de Alagoas. Rev. Bras. Ginecol. Obstet. 2008; 30(9):445-451.

16. Rocha DS, Pereira Netto M, Priore SE, Lima NMM, Rosado LEFPL, Franceschini SCC. Estado nutricional e anemia ferropriva em gestantes: relação com o peso da criança ao nascer. Rev. Nutr. 2005; 18(4):481-489.

17. Miglioli TC, Brito AM, Lira PIC, Figueroa JN, Batista Filho M. Anemia no binômio mãe-filho no Estado de Pernambuco, Brasil. Cad. Saúde Pública 2010; 26(9):1807-1820.

18. Brasil. Ministério da Saúde. Secretaria de Atenção à Saúde. Atenção ao pré-natal de baixo risco. Brasília: Ministério da Saúde; 2012. 318 p. Cadernos de Atenção Básica, no 32.

19. Totti HKSB, Zimmermmann JB, Pena DMF, Pereira MP, Bittencourt C, Coutinho T. Frequência de anemia e valores de normalidade para a hemoglobina em gestantes. HU Rev. 2009; 35(4):282-286.

20. Silva LSV, Thiapó AP, Souza GG, Saunders C, Ramalho A. Micronutrientes na gestação e lactação. Rev. Bras. Saúde Mater. Infant. 2007; 7(3):237-244.

21. Américo SCM, Ferraz, FN. Prevalência de anemias em gestantes do município de Campo Mourão PR entre os períodos de 2005 a 2008. Semina Cienc. Biol. Saúde 2011; 32(1):59-68.

Recebido: 16/9/2015

Revisado: 18/11/2015

Aceito: 01/3/2016 